\title{
Modeling Anomalous Hysteresis in Perovskite Solar Cells
}

\author{
Stephan van Reenen, Martijn Kemerink and Henry J. Snaith
}

\section{Linköping University Post Print}

\section{Tweet}

N.B.: When citing this work, cite the original article.

Original Publication:

Stephan van Reenen, Martijn Kemerink and Henry J. Snaith, Modeling Anomalous Hysteresis in Perovskite Solar Cells, 2015, Journal of Physical Chemistry Letters, (6), 19, 3808-3814. http://dx.doi.org/10.1021/acs.jpclett.5b01645

Copyright: American Chemical Society

http://pubs.acs.org/

Postprint available at: Linköping University Electronic Press

http://urn.kb.se/resolve?urn=urn:nbn:se:liu:diva-122432 


\section{Modelling anomalous hysteresis in perovskite solar}

\section{cells}

Stephan van Reenen, ${ }^{\dagger}$ Martijn Kemerink, ${ }^{\ddagger}$ and Henry J. Snaith ${ }^{\dagger} *$

†Department of Physics, University of Oxford, Clarendon Laboratory, Parks Road, Oxford, OX1 3PU, UK.

Department of Physics, Chemistry and Biology (IFM), Linköping University, SE-58 183, Linköping, Sweden.

\section{Corresponding Author}

*E-mail: h.snaith1@physics.ox.ac.uk

Organic-inorganic lead halide perovskites are distinct from most other semiconductors, since they exhibit characteristics of both electronic and ionic motion. Accurate understanding of the optoelectronic impact of such properties is important to fully optimize devices and be aware of any limitations of perovskite solar cells and broader optoelectronic devices. Here we use a numerical drift-diffusion model to describe device operation of perovskite solar cells. To achieve hysteresis effects in the modelled current-voltage characteristics, we must include both ion migration and electronic charge traps, serving as recombination centers. Trapped electronic charges recombine with oppositely charged free electronic carriers, of which the density depends on the bias-dependent ion distribution in the perovskite. Our results therefore show that reduction 
of either the density of mobile ionic species or carrier trapping at the perovskite interface will remove the adverse hysteresis effects in perovskite solar cells, giving a clear target for ongoing research effort and unifying previously conflicting experimental observations and theories.

\section{TOC GRAPHICS}

\section{Perovskite solar cells}
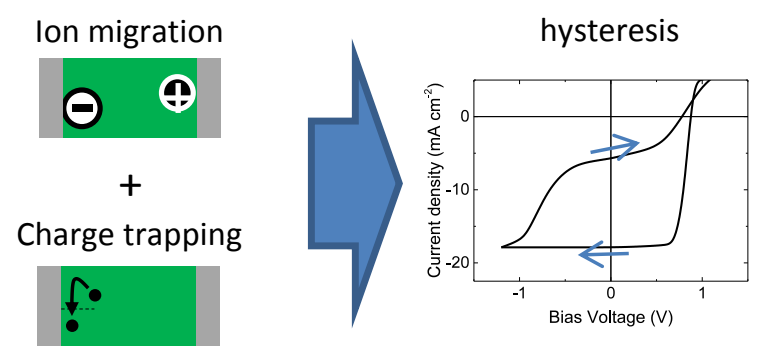

KEYWORDS perovskite; photovoltaics; hysteresis; trapping; ions

\section{TEXT}

Research interest in organic-inorganic lead halide perovskite semiconductors has risen tremendously within the last 6 years due to their use in photovoltaics, already giving rise to power conversion efficiencies of over $20 \%{ }^{1-6}$ Despite this tremendous growth, knowledge of the perovskite material properties itself and the relevant physical processes is still lacking. Currently, one of the major issues holding back development of perovskite solar cells is the presence of hysteresis in the voltage-dependent photocurrent. ${ }^{7-10}$ Even solar cells exhibiting over $20 \%$ efficiency exhibit hysteresis, and the efficiency is determined by taking the average of the forward and reverse scans, or by holding the cell close to the maximum power point until 
stabilised steady-state power output is achieved. The physical origin of this hysteresis has been linked to a large variety of processes like i) trapping of electronic carriers at the perovskite interface(s); ${ }^{11-12}$ ii) ionic displacement; ${ }^{13}$ or iii) ferroelectric effects. ${ }^{14-15}$ Experimental work supports these theories as for instance surface passivation of perovskites has led to a reduction in trap density and simultaneous hysteresis. ${ }^{11,} 16$ On the other hand, hysteresis has been shown convincingly to occur at timescales corresponding to ionic transport. ${ }^{13}$ Nevertheless, no clear and complete description exists of how such processes can eventually lead to hysteresis in these semiconductors, while simultaneously considering all currently known processes governing device operation.

In this Letter, we use numerical modelling to investigate solar cell current voltage characteristics under light and dark where the photoactive material includes both mobile ionic species and electronic trap sites. We show that hysteresis effects in perovskite solar cells originate from a combination of ionic transport throughout the perovskite and trapping of electronic carriers at the interface. Depending on the (history of the) biasing conditions, this combination can result in a significant loss in photocurrent due to trap-assisted recombination at the perovskite charge collection layer interface. This relatively high rate of recombination at the interface is sustained by large electron and hole densities due to i) electronic compensation of ionic space charge and ii) electronic traps. We could not reproduce any hysteresis via ionic transport or electronic traps alone, checked by calculations covering the reasonable limits of relevant parameters. Only when assuming an extraction barrier between the perovskite and its neighbouring transport layer, could we "induce" hysteresis effects by including ionic transport alone, while excluding electronic traps. However, in this latter instance, hysteresis in open-circuit voltage that is commonly 
observed in experiments was not reproduced. This indicates that an extraction barrier can "amplify" hysteresis, but is not its primary cause.

The energy level diagram of the modelled perovskite solar cell device is shown in Figure 1. We use a numerical drift-diffusion model that was previously used in $\left[{ }^{17-18}\right]$ to successfully describe the operation of light-emitting electrochemical cells, in which ionic and electronic transport are highly relevant. Here, the model describes operation of typical perovskite solar cells including generation, recombination, transport, trapping, and injection of electronic and ionic charge carriers by calculating the drift-diffusion and displacement currents by forward integration in time of the continuity equations for electrons, holes, and ions. We determine the electrostatic potential by Poisson's equation. We include more details on the numerical model in the Supplementary Information.

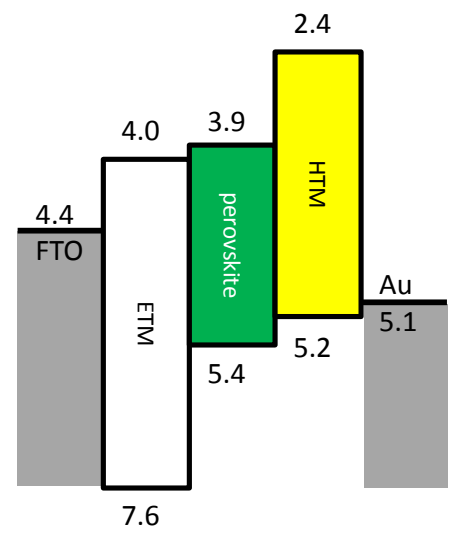

Figure 1. Energy level diagram in $\mathrm{eV}$ of the simulated perovskite solar cell.

Here follow the parameters we used to model a perovskite solar cell: A grid was used with $4 \mathrm{~nm}$ grid-point spacing. Model parameters for the electron transport material (ETM), the perovskite, and the hole transport material (HTM) are shown in Table 1. For the perovskite, an equal 
electron $\left(\mu_{\mathrm{n}}\right)$ and hole $\left(\mu_{\mathrm{p}}\right)$ mobility was chosen, similar to literature values. ${ }^{19-20}$ The dielectric constant $\varepsilon_{\mathrm{r}}$ was set at $6.5 .{ }^{21} \mathrm{~A}$ weakened form of Langevin recombination was implemented via a prefactor $L_{\mathrm{pre}}=10^{-5}$ in the recombination rate equation, following estimates from experiment. ${ }^{22}$ Homogeneous generation $G$ of free electrons and holes was assumed throughout the perovskite layer of $G=0$ or $2.5 \mathrm{~nm}^{-3} \mathrm{~s}^{-1}$ to model dark and light conditions respectively. ${ }^{23}$ The properties of the HTM and ETM were chosen such that these resembled doped materials like $\mathrm{TiO}_{2}$ and spiroOMeTAD.${ }^{19}$ Doping in these transport layers was modelled by a homogeneous density of immobile anions $(a)$ or cations $(c)$ which are electrostatically compensated by mobile electrons (n) and holes $(p)$.

Table 1. General device model parameters.

\begin{tabular}{lcccc}
\hline & & ETM & Perovskite & HTM \\
property & unit & & & \\
$\mu_{\mathrm{p}^{\prime}} \mu_{\mathrm{n}}$ & $\mathrm{cm}^{2} \mathrm{~V}^{-1} \mathrm{~s}^{-1}$ & 0.02 & 2 & 0.02 \\
$\varepsilon_{\mathrm{r}}$ & - & 80 & 6.5 & 3 \\
$L_{\text {pre }}$ & - & 1 & $10^{-5}$ & 1 \\
$G$ & $\mathrm{~nm}^{-3} \mathrm{~s}^{-1}$ & 0 & 2.5 & 0 \\
initial $a$ & $\mathrm{~cm}^{-3}$ & 0 & $\mathrm{X}$ & $8 \cdot 10^{17}$ \\
initial $c$ & $\mathrm{~cm}^{-3}$ & $8 \cdot 10^{17}$ & $\mathrm{X}$ & 0 \\
Initial $n$ & $\mathrm{~cm}^{-3}$ & $8 \cdot 10^{17}$ & 0 & 0 \\
initial $p$ & $\mathrm{~cm}^{-3}$ & 0 & 0 & $8 \cdot 10^{17}$ \\
Layer thickness & $\mathrm{nm}$ & 48 & 472 & 48 \\
\hline \multicolumn{4}{r}{} \\
\hline
\end{tabular}

In various publications hysteresis in perovskite solar cells has been attributed to the movement of ions throughout the perovskite: ${ }^{13}$ bias dependent ionic redistribution results in the build-up of ionic space charge at the interfaces of the perovskite, leading to a change in the built-in voltage 
of the perovskite. ${ }^{13}$ Such a change in built-in voltage may lead to different $I-V$ characteristics, dependent on the bias history before the actual bias sweep.

To determine whether mobile ions alone can indeed cause hysteresis, we attempted to replicate the experiment reported by Tress $e t$ al. ${ }^{13}$, which accurately shows the presence of hysteresis in perovskite solar cells. In their experiment, the perovskite solar cell was allowed to stabilize at varying bias voltages ( $V_{\text {stabilized }}$ ) prior to performing a fast $I-V$ sweep. The stabilization allows the ions to redistribute. The subsequent $I-V$ sweep is sufficiently fast to not allow ions to redistribute again during the sweep. In our model we reproduced this experiment by initially setting a homogeneous density of ions $a=c=10^{18} \mathrm{~cm}^{-3}$ in the perovskite, assuming only mobile anions with a mobility $\mu_{\mathrm{a}}=10^{-1} \mathrm{~cm}^{2} \mathrm{~V}^{-1} \mathrm{~s}^{-1}, V=V_{\text {stabilized, }}$, and $G=0$. Furthermore, we blocked ions at the interfaces, preventing ion exchange with the ETM or HTM. The consequence of this is that the quasi-steady state ion distribution is not affected by the ion mobility ${ }^{24}$ After the device reaches steady-state after roughly $3 \cdot 10^{-8} \mathrm{~s}$, we obtain the potential profiles which we show in Figure 2a. The potential drops at the perovskite interfaces prove the presence of ionic space charge screening the bulk of the perovskite from the externally applied electric field. Next, we set the anion mobility back to 0 to fix the ion distribution in the perovskite. This allows us to perform $I$ $V$ sweeps without ion redistribution or any need for knowledge on the ion mobility. In Figure $2 \mathrm{~b}$ we show the potential profiles at $V=0 \mathrm{~V}$ and $G=0$ to show the modified built-in voltage of the perovskite due to the induced ion distribution. In Figure $2 \mathrm{c}$ and $2 \mathrm{~d}$, respectively, we show the potential profiles and electron and hole density profiles at $V=0 \mathrm{~V}$ and $G=2.5 \mathrm{~nm}^{-3} \mathrm{~s}^{-1}$. The subsequent $I-V$ characteristics, during which the ions were immobile at different values of $V_{\text {stabilized }}$ and $G=2.5 \mathrm{~nm}^{-3} \mathrm{~s}^{-1}$, are plotted in Figure 3a. We observe that the modelled $I-V$ characteristics are all similar and thus independent of $V_{\text {stabilized. }}$ This result is in contrast with 
expectations and the experiments ${ }^{13}$ in which significant differences between $I-V$ characteristics with varying $V_{\text {stabilized }}$ are reported.
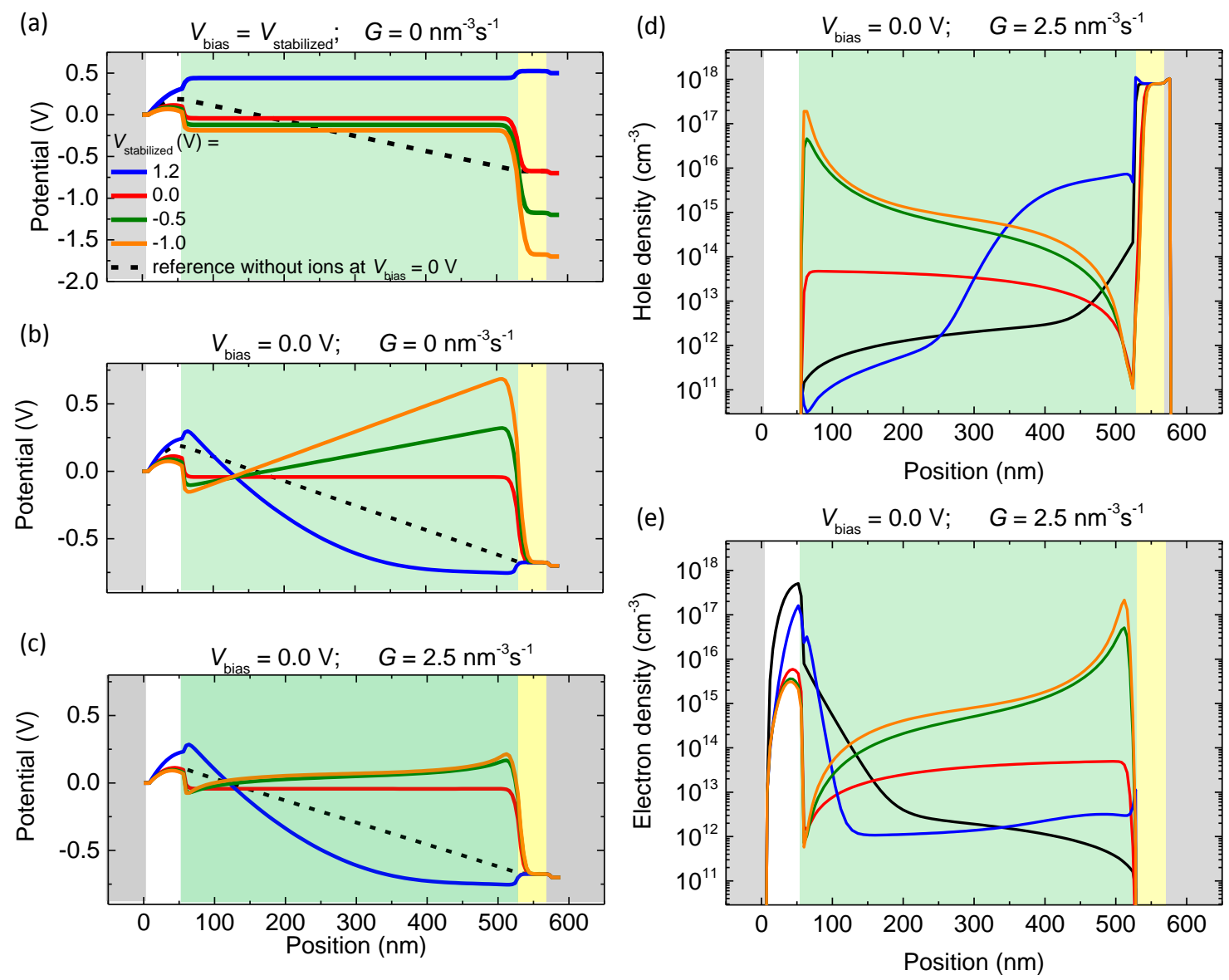

Figure 2. Modelled potential, electron, and hole density profiles for a typical perovskite device comprising ions but no traps. The shading in the background shows the device structure with from left to right: FTO, ETM, perovskite, HTM, Au. Electric field profiles can be found in the supplementary information. 
(a)

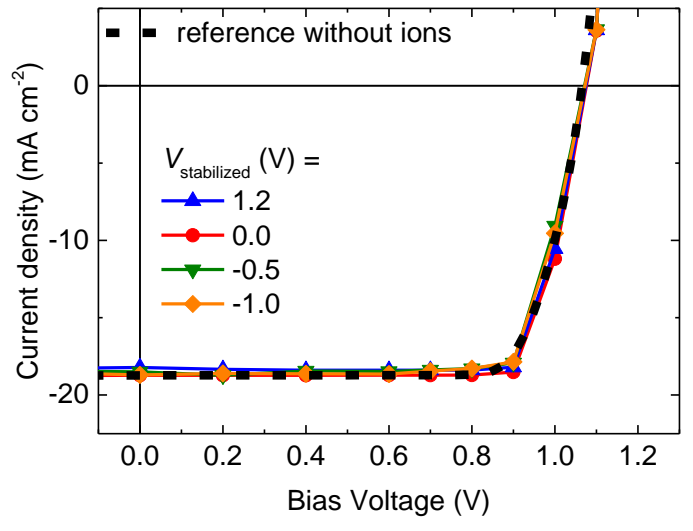

(b)

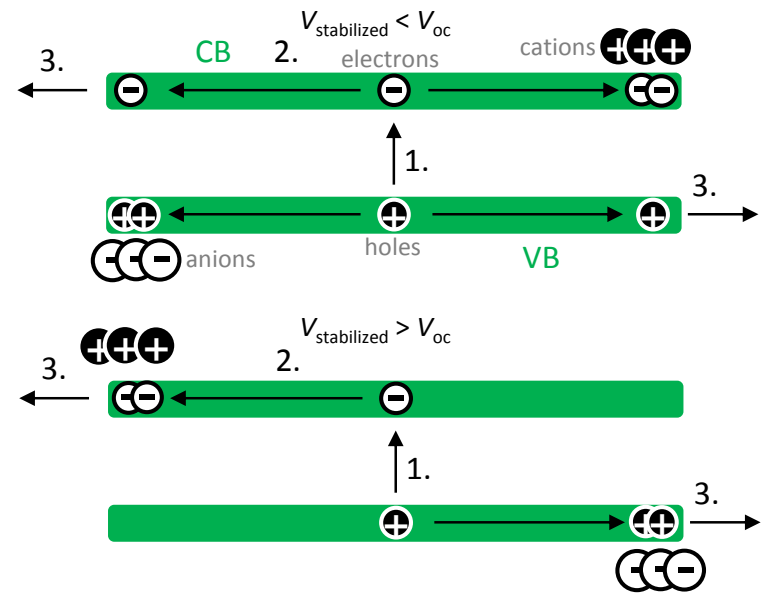

Figure 3. $J-V$ characteristics and interpretation of a perovskite solar cell with ions showing no hysteresis effects. (a) Modelled $J-V$ characteristics of a perovskite device comprising ions but no traps. (b-c) Schematics of the charge flow in the conduction (CB) and valence band (CV) of the perovskite in case (b) $V_{\text {stabilized }}<V_{\text {oc }}$ and (c) $V_{\text {stabilized }}>V_{\text {oc }}$ with 1. charge generation; 2. charge transport; 3. charge extraction.

We can attribute the absence of any hysteretic effect in the $I-V$ curves in Figure 3 a for different ion distributions, to electrostatic compensation (compare Figure $2 \mathrm{~b}$ and $\mathrm{c}$ ) of the ionic space charge by photogenerated electrons and holes. Close inspection of the modelling results shows a strong variation of the electron and hole densities (see Figure $2 \mathrm{~d}$ ) for varying $V_{\text {stabilized. For }}$ $V_{\text {stabilized }}=0,-0.5$ and $-1.0 \mathrm{~V}$, negative (positive) ionic space charge is formed at the interface between the ETM (HTM) and the perovskite. This negative (positive) ionic space charge is partially compensated by holes (electrons) in case $V_{\text {bias }}>V_{\text {stabilized. For }} V_{\text {stabilized }}=1.2 \mathrm{~V}$, however, the ion distribution is reversed: positive (negative) ionic space charge sits at the interface with the ETM (HTM). Again the negative (positive) space charge is partially compensated by holes 
(electrons). The schematics in Figure $3 \mathrm{~b}$ and $\mathrm{c}$ summarize this charge compensation mechanism and the flow of charge in the modelled perovskite solar cell including ion motion.

Since these results appear to contradict mounting experimental evidence pointing towards mobile ions being primarily responsible for hysteresis, we performed more simulations for varying initial ion densities in the perovskite, different types of mobile ions, and the inclusion of an extraction barrier between the perovskite and the ETM (see Supplementary information). We do not observe hysteresis effects in the $I-V$ characteristics upon variation of the total ion density. The results are also independent of the type of ionic carrier that is mobile, e.g. anions, cations, or both. In any of these three scenarios the mobile ions are able to generate ionic space charge at both of the perovskite interfaces by a combination of ion accumulation and depletion. When we include a significant extraction barrier, i.e. $0.3 \mathrm{eV}$ between the perovskite and the ETM conduction bands, we do observe hysteresis effects (see Supplementary Information). However, we only observe hysteresis in the "fill-factor" of the $I-V$ curve (the fill factor describes how sharp the bend is in the $I-V$ curve), while $V_{\text {oc }}$ and $J_{\text {sc }}$ remain intact. The hysteresis effects originate from a reduced (increased) charge extraction under working conditions when $V_{\text {stabilized }}=-1.0 \mathrm{~V}$ $\left(V_{\text {stabilized }}=1.6 \mathrm{~V}\right)$ by the induced negative (positive) ionic space charge at the ETM/perovskite interface. The reduced charge extraction results in a pile up of charge carriers in the bulk of the perovskite, enhancing recombination. For $V_{\text {stabilized }}=1.6 \mathrm{~V}$ the induced positive ionic space charge at the ETM/perovskite interface compensates the effect of the poor extraction barrier, resulting in lower charge carrier densities in the bulk of the perovskite and hence a reduced recombination. The key observation we make here is that the modelled hysteresis does not resemble the hysteresis observed in experiments by Tress et al. nor do their experiments show an S-shaped $I-V$ characteristic around $V=V_{\text {oc }}$, which we observe in our modelled result. This 
indicates that current and voltage hysteresis in perovskite devices must be explained by an alternative mechanism. In hind sight, we would not expect a modulation of the resistivity of a charge extraction barrier to induce voltage hysteresis, but simply induce a more or less pronounced s-shape of the $I-V$ curve as our model predicts. ${ }^{25-26}$

Besides mobile ions, it is also known that there is a non-negligible trap density in these perovskite materials. Hence, we now also consider interfacial electronic traps as the origin of hysteresis. At first we will consider only interfacial traps in the perovskite at the ETM/perovskite interface (see Supplementary Information), and neglect mobile ionic species. Following literature, we take an interfacial trap state density $N_{\mathrm{t}}=10^{17} \mathrm{~cm}^{-3}$ at the grid point in the perovskite which contacts the electron transport layer, with a trap energy $E_{\text {trap }}=0.5 \mathrm{eV} .{ }^{19} \mathrm{We}$ describe the trapping rate $\left(\right.$ in $\left.\mathrm{m}^{3} \mathrm{~s}^{-1}\right)$ by the product of the available trap density $\left(N_{\mathrm{t}}-n_{\mathrm{t}}\right)$, the free electron density $n$ at the same grid-point, and a trapping coefficient $c_{\mathrm{n}} . c_{\mathrm{n}}$ can be approximated by the product of the site volume (i.e. $n_{\operatorname{DOS}}{ }^{-1}=1 / 2.2 \cdot 10^{24}=5 \cdot 10^{-25} \mathrm{~m}^{3}$ following $\left[{ }^{19}\right]$ ) and the attempt frequency which can be approximated by a typical phonon frequency $\left(10^{10}-10^{12} \mathrm{~s}^{-1}\right)$. Based on these values, we used $c_{\mathrm{n}}=6 \cdot 10^{-13} \mathrm{~m}^{3} \mathrm{~s}^{-1}$ for the simulations.

We model trap-assisted recombination by a Langevin recombination rate of immobile trapped electrons and free holes, which is described by the product of the trapped electron density, the free hole density at the same grid-point and a recombination coefficient $c_{\mathrm{p}}$ (see Supplementary Information) ${ }^{27}$ Following Langevin recombination and the material parameters of the perovskite used in the model, we calculated $c_{\mathrm{p}}$ to be equal to $6 \cdot 10^{-13} \mathrm{~m}^{3} \mathrm{~s}^{-1}$.

In Figure 4a (dashed line) we show the modelled $I-V$ characteristic of a perovskite solar cell comprising electron traps at the ETM/perovskite interface without any mobile ions. We 
performed the calculations with the same parameters as described above. The current stabilizes at each voltage point within $10^{-5} \mathrm{~s}$, which directly shows that such interfacial trapping, by itself, is not the cause of hysteresis occurring at $>\mathrm{ms}$ timescales.

(a)

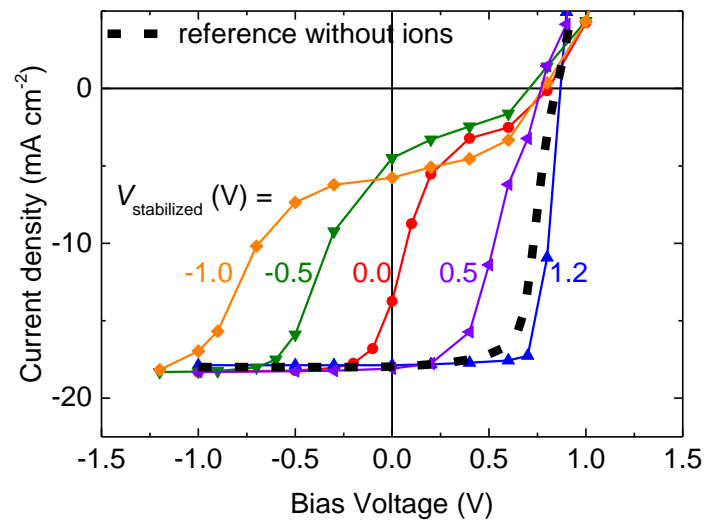

(b)

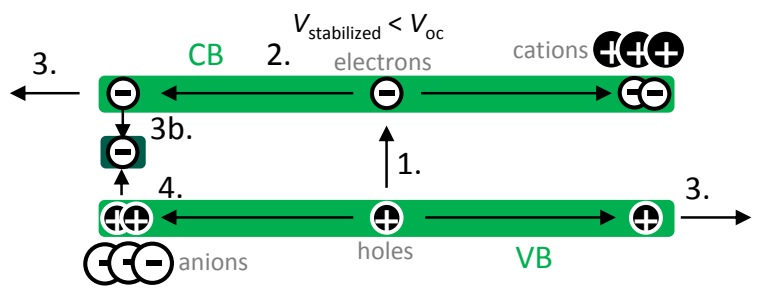

(c)

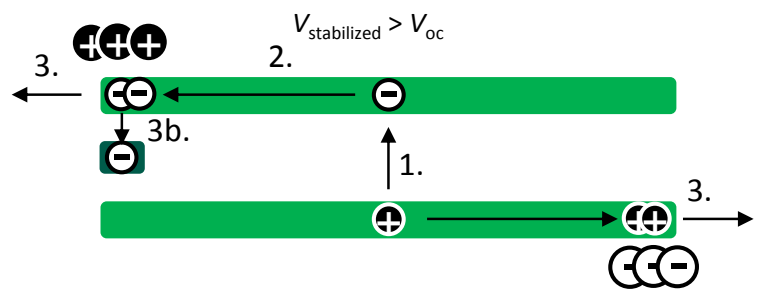

Figure 4. $J-V$ characteristics and interpretation of a perovskite solar cell with ions and traps showing hysteresis effects. (a) Modelled $J-V$ characteristics of a perovskite device comprising ions and traps. (b-c) Schematics of the charge flow in the conduction (CB) and valence band (CV) of the perovskite in case (b) $V_{\text {stabilized }}<V_{\mathrm{oc}}$ and (c) $V_{\text {stabilized }}>V_{\mathrm{oc}}$ with 1. charge generation; 2. charge transport; 3a. charge extraction; 3b. charge trapping; 4. trap-assisted charge recombination.

Since neither mobile ions, nor trap-assisted recombination alone can accurately reproduce the observed hysteresis effects, we seek to determine their combined effect. We repeat the modelling experiment where ions were allowed to stabilize at varying values of $V_{\text {stabilized }}$ in dark conditions before being immobilized, with the presence of traps. In Figure 4a we show the subsequent $I-V$ 
characteristics for $G=2.5 \mathrm{~nm}^{-3} \mathrm{~s}^{-1}$ in the perovskite, where we observe a clear dependence of the

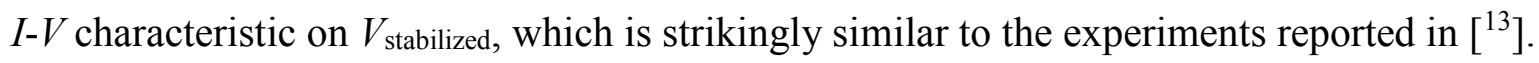

We can explain the results in Figure 4a by a loss in photocurrent due to recombination in the perovskite of free holes with trapped electrons at the ETM/perovskite interface, as we illustrate in Figure $4 \mathrm{~b}$ and c. For this loss to be significant, relatively large densities of free holes and trapped electrons are required. The trapped electron density is limited by the trap state density, i.e. $10^{17} \mathrm{~cm}^{-3}$ in this simulation, as well as the trapping rate relative to the recombination rate. The free hole density, shown in Figure 2 d, depends strongly on $V_{\text {stabilized }}$ over roughly 6 orders of magnitude: We observe relatively large hole densities at the ETM/perovskite interface for $V_{\text {bias }}>$

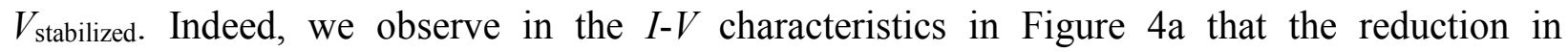
photocurrent takes place when $V_{\text {bias }}>V_{\text {stabilized. }}$ We undertook further modelling, where we observe that this loss of photocurrent can be reduced by reduction of the trapping coefficient, the trap state density, and the recombination coefficient (see Supplementary Information).

We have shown above that the combination of mobile ions and electron traps at the ETM/perovskite interface can lead to hysteresis effects which accurately reproduces experimental observations, for a reasonable choice of material parameters. We did, however, consider alternative combinations to test the boundaries of hysteresis inducing factors. For instance, if we consider mobile ions combined with hole traps at the perovskite/HTM interface, we can also reproduce hysteresis effects as the description is physically identical to the case we have described above. Moreover we also tested the combination of bulk traps and mobile ions (see Supplementary Information section E). Here we found that substitution of interface traps for bulk traps can result in similar hysteresis effects. To do so however requires the assumption of relatively large bulk trap state densities as well as relatively high recombination coefficients. 
These results show that to reduce hysteresis in perovskite solar cells, and more importantly to improve their efficiency, it is required to reduce either the density of mobile ionic species or charge trapping. Reduction of the trap density in our model indeed reduces the hysteresis effect (see Supplementary Information). This is in line with recent experimental work in which hysteresis has been reduced by passivation of traps at the perovskite interfaces. ${ }^{11-12,} 16,28$ Furthermore, the trap density at the interface is very likely to depend on the type of charge transport layers connecting the perovskite, ${ }^{29-30}$ and the quality and structure of the perovskite itself, ${ }^{31-32}$ which can strongly vary for different deposition methods. In addition, doping of the perovskite near the interfaces is expected to strongly influence the charge carrier profiles and hence the hysteresis mechanism described in this letter. ${ }^{33}$ This could lead to a hysteresis-free cell with large interfacial trap densities.

We have shown that anomalous hysteresis effects in perovskite solar cells can be understood as the combined effect of ion migration through the perovskite and trapping of charge carriers at the perovskite interfaces. Both ion migration and charge trapping can cause large densities of electrons and holes at the perovskite interface, with the relative density dependent on the electronic bias history, trap density and ionic density. For unfavourable biasing, a large density of trapped electrons and free holes accumulate at the perovskite ETM interface, inducing strong non-radiative trap-assisted recombination. The consequently large (non-radiative) recombination rate reduces the photocurrent and thereby the efficiency of the device. For favourable biasing a low density of trapped electrons and free holes accumulates at this interface, good for more ideal solar cell operation. Ion migration and charge trapping alone cannot reproduce hysteresis effects. Hence, reduction of hysteresis and simultaneous improvement of efficiency should be achievable by reducing either the density of mobile ionic species or electronic defects responsible for trap- 
assisted recombination. Importantly, the new coherent insight we have derived from this model, consolidates the previously perceived to be conflicting observations of hysteresis effects originating from different phenomena. We also note that it is not necessary to induce any consideration of ferroelectric effects to explain the observable hysteresis.

Although our model proves that interfacial traps and ion transport are both crucial to understand hysteresis effects in perovskite solar cells, it does not give any detail on the actual origin of the traps or the mechanism of ion transport throughout the perovskite. Hence to reduce hysteresis by reducing ion transport and interfacial traps, more research is required to gain more insight in these processes. Nevertheless, this model predicts that targeting these issues will lead to "ideal" solar cell operation.

More broadly, these results show that the presence of both mobile ions and traps in semiconductors can give rise to hysteretic effects that reduce performance in electronic devices. Therefore these results can be extended to other material systems like organic semiconductors, ${ }^{34}$ ionic transition metal complexes, ${ }^{35}$ or metal oxides, ${ }^{36}$ and they represent a sound platform for describing the operation of future devices.

\section{ASSOCIATED CONTENT}

Supporting Information. Computational methods. Additional computational results. This material is available free of charge via the Internet at http://pubs.acs.org.

\section{Corresponding Author}

*Email: h.snaith1@physics.ox.ac.uk 


\section{Notes}

The authors declare no competing financial interests.

\section{ACKNOWLEDGMENT}

We would like to thank the Nederlandse Organizatie voor Wetenschappelijk Onderzoek (NWO) for funding. This work was part funded by EPSRC.

\section{REFERENCES}

1. Kojima, A.; Teshima, K.; Shirai, Y.; Miyasaka, T., Organometal Halide Perovskites as Visible-Light Sensitizers for Photovoltaic Cells. J. Am. Chem. Soc. 2009, 131, 6050-6051.

2. Lee, M. M.; Teuscher, J.; Miyasaka, T.; Murakami, T. N.; Snaith, H. J., Efficient Hybrid Solar Cells Based on Meso-Superstructured Organometal Halide Perovskites. Science 2012, 338, 643-647.

3. Burschka, J.; Pellet, N.; Moon, S. J.; Humphry-Baker, R.; Gao, P.; Nazeeruddin, M. K.; Gratzel, M., Sequential deposition as a route to high-performance perovskite-sensitized solar cells. Nature 2013, 499, 316-319.

4. Liu, M. Z.; Johnston, M. B.; Snaith, H. J., Efficient planar heterojunction perovskite solar cells by vapour deposition. Nature 2013, 501, 395-398.

5. Jeon, N. J.; Noh, J. H.; Yang, W. S.; Kim, Y. C.; Ryu, S.; Seo, J.; Seok, S. I., Compositional engineering of perovskite materials for high-performance solar cells. Nature 2015, 517, 476-480.

6. Yang, W. S.; Noh, J. H.; Jeon, N. J.; Kim, Y. C.; Ryu, S.; Seo, S.; Seok, S. I., Highperformance photovoltaic perovskite layers fabricated through intramolecular exchange. Science 2015, 348, 1234-1237.

7. Jena, A. K.; Chen, H.-W.; Kogo, A.; Sanehira, Y.; Ikegami, M.; Miyasaka, T., The Interface between FTO and the TiO2 Compact Layer Can Be One of the Origins to Hysteresis in Planar Heterojunction Perovskite Solar Cells. Acs Appl. Mater. Inter. 2015, 7, 9817-9823. 
8. Snaith, H. J.; Abate, A.; Ball, J. M.; Eperon, G. E.; Leijtens, T.; Noel, N. K.; Stranks, S. D.; Wang, J. T. W.; Wojciechowski, K.; Zhang, W., Anomalous Hysteresis in Perovskite Solar Cells. J. Phys. Chem. Lett. 2014, 5, 1511-1515.

9. Kim, H. S.; Park, N. G., Parameters Affecting I-V Hysteresis of CH3NH3PbI3 Perovskite Solar Cells: Effects of Perovskite Crystal Size and Mesoporous TiO2 Layer. J. Phys. Chem. Lett. 2014, 5, 2927-2934.

10. Liu, C.; Fan, J.; Zhang, X.; Shen, Y.; Yang, L.; Mai, Y., Hysteretic Behavior upon Light Soaking in Perovskite Solar Cells Prepared via Modified Vapor-Assisted Solution Process. Acs Appl. Mater. Inter. 2015, 7, 9066-9071.

11. Shao, Y. H.; Xiao, Z. G.; Bi, C.; Yuan, Y. B.; Huang, J. S., Origin and elimination of photocurrent hysteresis by fullerene passivation in $\mathrm{CH} 3 \mathrm{NH} 3 \mathrm{PbI} 3$ planar heterojunction solar cells. Nat. Commun. 2014, 5, 5784.

12. Xu, J.; Buin, A.; Ip, A. H.; Li, W.; Voznyy, O.; Comin, R.; Yuan, M.; Jeon, S.; Ning, Z.; McDowell, J. J.; Kanjanaboos, P.; Sun, J.-P.; Lan, X.; Quan, L. N.; Kim, D. H.; Hill, I. G.; Maksymovych, P.; Sargent, E. H., Perovskite-fullerene hybrid materials suppress hysteresis in planar diodes. Nat. Commun. 2015, 6, 7081.

13. Tress, W.; Marinova, N.; Moehl, T.; Zakeeruddin, S. M.; Nazeeruddin, M. K.; Gratzel, M., Understanding the rate-dependent J-V hysteresis, slow time component, and aging in $\mathrm{CH} 3 \mathrm{NH} 3 \mathrm{PbI} 3$ perovskite solar cells: the role of a compensated electric field. Energ Environ Sci 2015, 8, 995-1004.

14. Wei, J.; Zhao, Y. C.; Li, H.; Li, G. B.; Pan, J. L.; Xu, D. S.; Zhao, Q.; Yu, D. P., Hysteresis Analysis Based on the Ferroelectric Effect in Hybrid Perovskite Solar Cells. J. Phys. Chem. Lett. 2014, 5, 3937-3945.

15. Frost, J. M.; Butler, K. T.; Walsh, A., Molecular ferroelectric contributions to anomalous hysteresis in hybrid perovskite solar cells. APL Mat. 2014, 2, 081506.

16. Heo, J. H.; You, M. S.; Chang, M. H.; Yin, W.; Ahn, T. K.; Lee, S.-J.; Sung, S.-J.; Kim, D. H.; Im, S. H., Hysteresis-less mesoscopic $\mathrm{CH} 3 \mathrm{NH} 3 \mathrm{PbI} 3$ perovskite hybrid solar cells by introduction of Li-treated TiO2 electrode. Nano Energy 2015, 15, 530-539.

17. Meier, S. B.; Van Reenen, S.; Lefevre, B.; Hartmann, D.; Bolink, H. J.; Winnacker, A.; Sarfert, W.; Kemerink, M., Dynamic doping in planar ionic transition metal complex-based light-emitting electrochemical cells. Adv. Funct. Mater. 2013, 23, 3531-3538.

18. van Reenen, S.; Janssen, R. A. J.; Kemerink, M., Fundamental Tradeoff between Emission Intensity and Efficiency in Light-Emitting Electrochemical Cells. Adv. Funct. Mater. 2015, 25, 3066-3073.

19. Minemoto, T.; Murata, M., Device modeling of perovskite solar cells based on structural similarity with thin film inorganic semiconductor solar cells. J. Appl. Phys. 2014, 116, 054505.

20. Stranks, S. D.; Eperon, G. E.; Grancini, G.; Menelaou, C.; Alcocer, M. J. P.; Leijtens, T.; Herz, L. M.; Petrozza, A.; Snaith, H. J., Electron-Hole Diffusion Lengths Exceeding 1 Micrometer in an Organometal Trihalide Perovskite Absorber. Science 2013, 342, 341-344.

21. Hirasawa, M.; Ishihara, T.; Goto, T., Exciton Features in 0-Dimensional, 2-Dimensional, and 3-Dimensional Networks of [Pbi6]4- Octahedra. J. Phys. Soc. Jpn. 1994, 63, 3870-3879.

22. Wehrenfennig, C.; Eperon, G. E.; Johnston, M. B.; Snaith, H. J.; Herz, L. M., High Charge Carrier Mobilities and Lifetimes in Organolead Trihalide Perovskites. Adv. Mater. 2014, $26,1584-1589$. 
23. Foster, J. M.; Snaith, H. J.; Leijtens, T.; Richardson, G., A Model for the Operation of Perovskite Based Hybrid Solar Cells: Formulation, Analysis, and Comparison to Experiment. Siam J Appl Math 2014, 74, 1935-1966.

24. van Reenen, S.; Matyba, P.; Dzwilewski, A.; Janssen, R. A. J.; Edman, L.; Kemerink, M., A Unifying Model for the Operation of Light-Emitting Electrochemical Cells. J. Am. Chem. Soc. 2010, 132, 13776-13781.

25. Nelson, J.; Kirkpatrick, J.; Ravirajan, P., Factors limiting the efficiency of molecular photovoltaic devices. Phys. Rev. B 2004, 69, 035337.

26. Tress, W.; Leo, K.; Riede, M., Influence of Hole-Transport Layers and Donor Materials on Open-Circuit Voltage and Shape of I-V Curves of Organic Solar Cells. Adv. Funct. Mater. 2011, 21, 2140-2149.

27. Kuik, M.; Koster, L. J. A.; Wetzelaer, G. A. H.; Blom, P. W. M., Trap-Assisted Recombination in Disordered Organic Semiconductors. Phys. Rev. Lett. 2011, 107, 256805.

28. Noel, N. K.; Abate, A.; Stranks, S. D.; Parrott, E. S.; Burlakov, V. M.; Goriely, A.; Snaith, H. J., Enhanced Photoluminescence and Solar Cell Performance via Lewis Base Passivation of Organic Inorganic Lead Halide Perovskites. ACS Nano 2014, 8, 9815-9821.

29. Kim, H. S.; Park, N. G., Parameters Affecting I-V Hysteresis of CH(3)NH(3)Pbl(3) Perovskite Solar Cells: Effects of Perovskite Crystal Size and Mesoporous TiO2 Layer (vol 5, pg 2927, 2014). J. Phys. Chem. Lett. 2014, 5, 3434-3434.

30. Choi, H.; Mai, C. K.; Kim, H. B.; Jeong, J.; Song, S.; Bazan, G. C.; Kim, J. Y.; Heeger, A. J., Conjugated polyelectrolyte hole transport layer for inverted-type perovskite solar cells. Nat. Commun. 2015, 6 .

31. Tripathi, N.; Yanagida, M.; Shirai, Y.; Masuda, T.; Han, L. Y.; Miyano, K., Hysteresisfree and highly stable perovskite solar cells produced via a chlorine-mediated interdiffusion method. J. Mater. Chem. A 2015, 3, 12081-12088.

32. Nie, W. Y.; Tsai, H. H.; Asadpour, R.; Blancon, J. C.; Neukirch, A. J.; Gupta, G.; Crochet, J. J.; Chhowalla, M.; Tretiak, S.; Alam, M. A.; Wang, H. L.; Mohite, A. D., Highefficiency solution-processed perovskite solar cells with millimeter-scale grains. Science 2015, $347,522-525$.

33. Leijtens, T.; Stranks, S. D.; Eperon, G. E.; Lindblad, R.; Johansson, E. M. J.; McPherson, I. J.; Rensmo, H.; Ball, J. M.; Lee, M. M.; Snaith, H. J., Electronic Properties of MesoSuperstructured and Planar Organometal Halide Perovskite Films: Charge Trapping, Photodoping, and Carrier Mobility. ACS Nano 2014, 8, 7147-7155.

34. Pei, Q. B.; Yu, G.; Zhang, C.; Yang, Y.; Heeger, A. J., Polymer Light-Emitting Electrochemical-Cells. Science 1995, 269, 1086-1088.

35. Slinker, J.; Bernards, D.; Houston, P. L.; Abruna, H. D.; Bernhard, S.; Malliaras, G. G., Solid-state electroluminescent devices based on transition metal complexes. Chem. Commun. 2003, 2392-2399.

36. Pavan, M.; Ruhle, S.; Ginsburg, A.; Keller, D. A.; Barad, H. N.; Sberna, P. M.; Nunes, D.; Martins, R.; Anderson, A. Y.; Zaban, A.; Fortunato, E., TiO2/Cu2O all-oxide heterojunction solar cells produced by spray pyrolysis. Sol. Energy Mater. Sol. Cells 2015, 132, 549-556. 
